\title{
Phytotherapie in der HNO-Praxis
}

\section{Schweizerische Tagung für Phytotherapie 2019}

Die Schweizerische Medizinische Gesellschaft für Phytotherapie SMGP darf auf eine weitere erfolgreiche Tagung zurückblicken. Mit Hilfe von vier verbandseigenen HNO-Fachärzten und erfahrenen Apothekerinnen, die sich an der Gestaltung des Programms beteiligten und teilweise selbst von ihren Erfahrungen berichteten, kam eine Veranstaltung zustande, die mit Authentizität überzeugte - vor allem deshalb, weil der Patient bei der Behandlung verschiedenster Symptome in den Mittelpunkt gestellt wurde. Fast 300 Teilnehmende waren mit dabei und füllten das Trafo in Baden fast bis auf den letzten Platz ( $\triangleright$ Abb. 1).

Das Thema „Phytotherapie in der HNOPraxis“ traf den Nerv der Zeit, passte dieses doch zur in der Woche durchgeführten Antibiotica-Awareness Week der WHO, zu der auch Veranstaltungen in der Schweiz stattgefunden haben. Gerade im HNO-Bereich sind viele Erkrankungen nicht bakteriell bedingt, auch wenn oft meist geht es darum, Sekundärinfekte zu verhindern - Antibiotika eingesetzt werden. Für die primär viral bedingten Erkrankungen gibt es viele phytotherapeutische Alternativen, die sich in der Praxis bewähren. Um zu entscheiden, ob Phytotherapie oder Antibiotika angebracht sind, braucht es eine klare Diagnose. Diese war dann auch das große Anliegen der beteiligten Referierenden.

\section{Virale Atemwegsinfektion / Prophylaxe und (Phyto)-Therapie}

Prof. Dr. med. Michael Tamm, Universitätsklink Basel. Mehr als zwei Drittel der weltweit verschriebenen Antibiotika betreffen die Behandlung von Atemwegsinfektionen. Infektionen der unteren Atemwege werden bei immunkompetenten Patienten grob in akute Bronchitiden, Exazerbationen bei Asthma und bei COPD sowie Pneumonien unterteilt. Unter

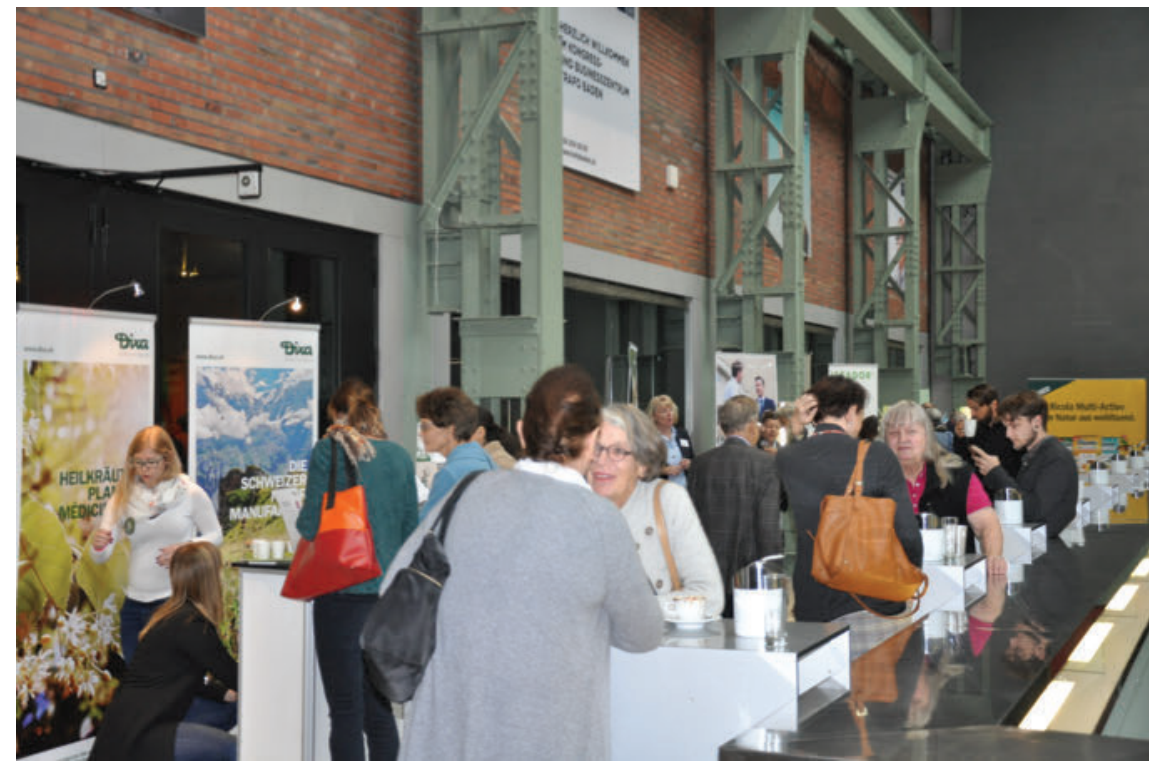

-Abb. 1 Gut besucht - lebhafter Austausch: die SMGP-Jahrestagung 2019 im „Trafo“ in Baden. Quelle: Patrick Fontana

speziellen Infektionen leiden Patienten mit einer zystischen Fibrose, mit einem Lungentransplantat oder mit Immundefekten. Eine akute Bronchitis ist in über 95\% der Fälle viral. Sie werden in unseren Breitengraden am häufigsten von Rhinoviren verursacht. Weitere wichtige Viren sind das Influenza-, das Corona-, das RSV, das Parainfluenza-, das Metapneumonie- und das Adenovirus. Interessanterweise sind die meisten Exazerbationen bei Asthma ebenfalls viral und nicht bakteriell bedingt. Für die Therapie ist die Unterscheidung wichtig, ob es sich um einen „banalen“ Virusinfekt oder eine Pneumonie handelt. Bei letzterer sind Antibiotika indiziert, beim banalen Virusinfekt dagegen unsinnig.

Hinweise für eine Pneumonie sind ein einseitiger Auskultationsbefund, Atemnot und Tachypnoe sowie das Anhalten des Fiebers über 4 Tage. Die Bestimmung des CRP und/oder ein Röntgenbild sowie die Bestimmung von Procalcitonin helfen bei der Unterscheidung viraler Infekt/bakterieller Infekt. Große randomisierte Studien zeigen, dass bei niedrigem CRP, resp. Procalcitonin ein Verzicht auf Antibiotika den
Ausgang der Infektion nicht beeinflusst. Ist einer der beiden Laborwerte erhöht, ist die Verabreichung eines Antibiotikums indiziert. Zu beachten ist, dass der CRPSpiegel erst $24 \mathrm{~h}$ nach Beginn des Infekts oder einer Sepsis ansteigt.

Das Lungenzentrum der Universitätsklinik empfiehlt eine Grippeimpfung für ältere Patienten und für Patienten mit Herzinsuffizienz, COPD, Diabetes etc. Eine Pneumokokken-Impfung empfiehlt das Zentrum für alle Patienten mit einer Lungenkrankheit und / oder einem Immundefekt.

Das phytotherapeutische Präparat EPs ${ }^{\circledR 7630 ~(E x t r a k t ~ a u s ~ P e l a r g o n i u m ~}$ sidoides) wirkt antiviral, antibakteriell und sekretomotorisch. Die antivirale Wirkung konnte an Zellkulturen nachgewiesen werden: Mit dem Rhinovirus infizierte Zellen überleben länger nach der Behandlung mit dem Präparat und die Replikation von Viruspartikeln wird verringert. Insgesamt befördern pflanzliche Arzneimittel bei der Behandlung von Atemwegsinfekten die Heilung und verkürzen die Dauer der Symptome. 


\section{Rhinosinusitis ohne}

\section{Antibiotika}

Dr. med. Kaspar Strub, Basel. Die Rhinosinusitis ist eine Entzündung der Nase und der Nasennebenhöhlen. Im Zentrum der Therapie steht das Wiedererlangen der normalen Funktion der Schleimhaut, der mukoziliären Clearance und Belüftung, und das, wenn immer möglich, ohne Antibiotika. Die Phytotherapie ist hier eine tragende Säule der Therapieoptionen.

Die Kenntnis der Besiedelung der Atemwege hat sich in den letzten Jahren stark verändert. Abstriche, Kultur, Mikroskopie oder auch Schnellteste erfassen nicht alle relevanten pathogenen Keime, z.B. anaerobe Keime oder Pilze. Ein weiterer Nachteil des klassischen Keimnachweises ist, dass das Ergebnis nicht den Zustand des Mikrobioms zum Zeitpunkt der Probennahme abbildet. Moderne PCR-Methoden erlauben es, Viren sehr rasch nachzuweisen. Ein wichtiger Keim bleibt immer noch außen vor: Staphylococcus aureus, der bei 20-30\% der beschwerdefreien Personen vorkommt. Die PCR-Methode ermöglicht es, die aktuelle Zusammensetzung eines Mikrobioms vollständig zu erfassen. Die Verwendung von Antibiotika führt zu einer anhaltenden Verschiebung im Mikrobiom, Es besteht die Gefahr, dass eine Resistenzentwicklung induziert wird. Mit wenigen Fragen und nichtinvasiven Untersuchungen lässt sich eine allergische Rhinosinusitis von einer nichtallergischen Rhinitis und Rhinosinusitis abgrenzen. Wichtig: Auf dem Boden einer Allergie können meist harmlose Rhinoviren schwere Asthma-Exazerbationen auslösen. Die Untersuchung erfolgt mit Otoskop, Mundspatel und einer Lichtquelle. Als „schmerzloses“ Endoskop steht das Laryngoskop zu Verfügung. Das konventionelle Röntgenbild hat weitgehend ausgedient.

- Therapie der ersten Wahl: Abschwellende Nasensprays oder -tropfen, hypertone $\mathrm{NaCl}$-Spülungen, topische Steroide, NSAR, eventuell Antihistaminika.

- Zur Entzündungshemmung: NSAR, Antihistaminika, Tesalin N (schweizerisches Arzneimittel aus Pestwurzextrakt).

- Phytotherapie: Sinupret, Bronchipret, Kaloba / Umckaloabo, Angocin, Gelodurat.

\section{Atemwegsinfekte bei \\ Kindern - neue \\ Präventionsstudie mit Echinacea}

PD. Dr. med. Werner Albrich, Kantonsspital St. Gallen. Die Einteilung erfolgt in obere und untere Atemwegsinfekte. Zu den oberen Atemwegsinfekten gehören der grippale Infekt (Erkältung), die Rhinosinusitis, Tonsillitis, Pharyngitis, Laryngitis und Otitis media, zu den unteren die Tracheitis, Bronchitis, Bronchiolitis und Pneumonie.

Namentlich Infekte der oberen Luftwege sind ausgesprochen häufig. Sie werden von mehr als 100 Viren oder ihren Subtypen verursacht, sodass es nicht möglich ist, gegen alle eine ausreichende Immunität aufzubauen. Infekte der oberen Luftwege betreffen vor allem kleine Kinder, die anatomische und immunologische Prädilektionen aufweisen, nehmen aber mit dem Alter ab. 10-15\% der Kinder können bis zu 12 Episoden im Jahr erleiden, ohne dass zwingend ein Immundefekt vorliegen muss. Solche Fälle machen in einer Kinderarztpraxis 40$60 \%$ der Konsultationen aus. Grippale Infekte sind eine typisch virale Erkrankung, Antibiotika sind nicht indiziert. Trotzdem betreffen bis zu 75-80\% der Antibiotikaverschreibungen einen Virusinfekt. Ärztliche
Kunst ist es, zu erkennen, ob ein solcher Infekt banal ist oder ob Zeichen für einen schweren Verlauf bestehen, der dann doch einer antibiotischen Behandlung bedarf. Besonders tückisch an den Atemwegsinfekten ist, dass sie ansteckend sind, bevor der betroffene Patient Symptome zeigt. Eine wirksame Prävention ist deshalb auch für die Kontaktpersonen der Kinder von großem Vorteil.

Extrakte aus Echinacea, in der Schweiz ist vor allem Echinacea purpurea im Einsatz ( Abb.2), sind antiviral, entzündungshemmend, immunmodulierend und antibakteriell wirksam. Bei guter Verträglichkeit und ohne Resistenzentwicklung reduzieren optimale Zubereitungen aus Echinacea purpurea die Dauer der Symptome einer Erkältung. Das belegte eine kürzlich durchgeführte klinische Studie. Total 202 Kinder aus Kinderarzt- oder Allgemeinarztpraxen im Alter von 4-12 Jahren erhielten während zweier Monate entweder $3 \times 1$ Echinaforce ${ }^{\circledR}$ Junior Tablette $(400 \mathrm{mg}$ Extrakt aus Kraut und Wurzel, $n=103$ ) oder $3 x$ täglich 1 Tablette Vitamin C (500 mg, $\mathrm{n}=98$ ). Nach 1 Woche Pause wurde die Behandlung nochmals für 2 Monate weitergeführt. Kinder, die Echinaforce $^{\circledR}$ erhielten, hatten weniger Erkältungstage (429 vs. 602, $\mathrm{p}<0,001$ ), um 32,5\% weniger Atemwegsinfekte, weniger Antibiotikagaben (6 Kinder für

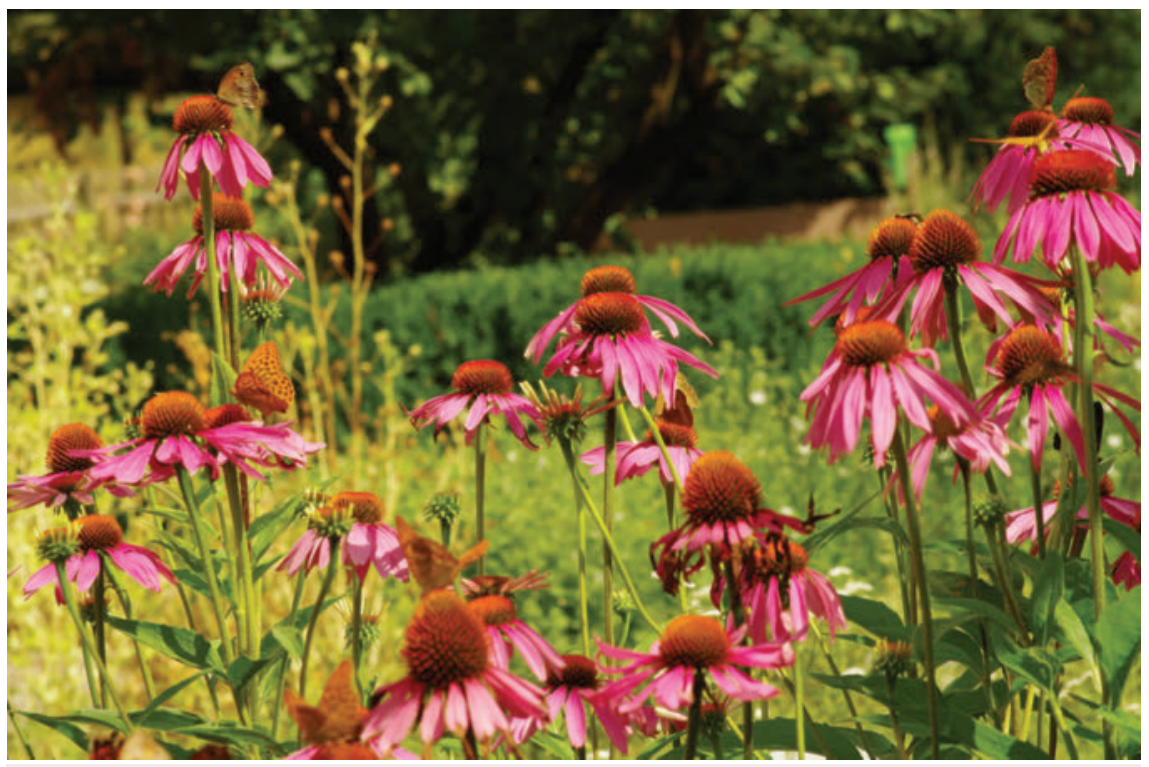

-Abb. 2202 Kinder zwischen 4 und 12 Jahren profitierten von einem Echinacea-Präparat signifikant stärker als von 1500 mg Vitamin C pro Tag. Quelle: Renate Stockinger / Thieme Gruppe 
insgesamt 45 Tage vs. 15 Kinder für insgesamt 216 Tage $(-76,3 \%, p<0,001)$, weniger bakterielle Superinfekte $(-65,0 \%$, $\mathrm{p}<0,05$ ), weniger Komplikationen (Pneumonie, Tonsillitis, Otitis media), weniger Fiebertage $(-67,3 \%$, teilweise mitverursacht durch Verkürzung der Krankheitsdauer). Fazit: Echinaforce ${ }^{\circledR}$ kann als Prophylaxe verabreicht sowohl beim Erwachsenen als auch beim Kind Atemwegsinfekte vermindern.

\section{Gehörgangsekzem und chronische Otitis media}

Dr. med. Stefan Schumacher, Winterthur. An einem durchschnittlichen Sprechstundentag sieht ein HNO-Arzt bei mindestens 5 Patienten ein Gehörgangsekzem. Es ist nicht immer so stark symptomatisch, dass es den Betreffenden stört. Mal ist es schuppend, mal juckend, mal nässend, oft aber auch nur gerötet. Das Ekzem befindet sich in der Regel am Übergang von der behaarten Haut zur Gehörgangshaut. Oft besteht eine Behinderung des Abflusses von Ohrenschmalz mit wiederkehrendem Cerumen. Dieses wiederum triggert das Ekzem. Es handelt sich nicht um eine schwerwiegende Störung, nervt aber die Betroffenen. Die Pathogenese wird noch nicht verstanden. Die Behandlung beschränkt sich auf ein Unterdrücken der Symptome, primär mit Kortisonpräparaten. Pflanzliche Präparate eignen sich vor allem zur Erhaltungstherapie. Schuppende Ekzeme können mit Olivenöl oder Mahonia-Präparaten (Mahonia aquifolium), nässende Ekzeme mit CardiospermumZubereitungen behandelt werden. Salben aus ätherischen Ölen sind als Erhaltungstherapie ebenfalls wirksam. Bewährt haben sich Kombinationen von primär entzündungshemmenden Ölen mit antiinfektiös wirksamen Ölen, z. B. Zitronengras/ Pelargonie oder Lavendel / Schafgarbe.

\section{Chronische Otitis externa beim Hund: Chancen für Phytotherapeutika}

Prof. PhD Petra Roosje, Bern. Im Hauptprogramm wurde auch ein Referat für die Veterinärmediziner, die am Nachmittag ein eigenes Programm hatten, präsentiert. Es ergab sich ein reizvoller Vergleich bei einem Symptomenkomplex, der Mensch und Haustier in ähnlicher Weise betrifft.

Otitis externa ist ein häufiges Problem des Hundes und betrifft rund $10 \%$ der Patienten einer Kleintierpraxis. Die Diversität der Hunderassen widerspiegelt sich in großen Unterschieden in der Form und Größe der Ohrmuschel, der Länge und des Volumens des äußeren Gehörgangs und in der Dichte der Behaarung an der Ohrmuschel und innerhalb des Gehörgangs. Rassehunde erhalten die Diagnose Otitis media häufiger als Mischlinge. Viele Hunde müssen nach der Diagnose wiederholt vorgestellt und behandelt werden. Vorstellungsgründe sind ein übler Geruch aus den Ohren, Austreten von größeren Mengen von Cerumen, ein purulentes Exsudat, vermehrt Kopfschütteln oder Kratzen an der Ohrbasis, Zeigen von Schmerz beim Berühren des Kopfs oder der Ohrmuschel. Primäre Ursachen sind Ektoparasiten, eine atopische Dermatitis, andere Hautkrankheiten, Futtermittelreaktionen, Fremdkörper, evtl. eine Otitis media. Sekundäre Ursachen sind bakterielle oder Hefepilzinfektionen sowie gemischte oder Prädisponierende Faktoren können anatomische Gegebenheiten sein, Umgebungsfaktoren (z. B. Feuchtigkeit), ein Trauma des Gehörgangs oder Obstruktionen (z. B. durch eine Neoplasie). Perpetuierende Faktoren sind progressive pathologische Veränderungen des Gehörgangs (hyperplastische Epidermis, zu kleines Lumen, Mineralisation des Knorpels) oder Veränderungen des Trommelfells.

Zur Diagnose sollte eine Kultur angelegt werden, ein Abstrich ist zu wenig aussagekräftig. Häufig werden opportunistisch pathogene Keime gefunden und auch Methicillin-resistente Salmonella pseudointermedius oder Pseudomonas aeruginosa. Sie sind gegen sämtliche in der Schweiz zugelassene veterinärmedizinische Ohrmedikamente, die ein Antibiotikum enthalten, resistent. Der Pilz, der am häufigsten beim Hund gefunden wird, ist Malassezia pachydermatis. $\mathrm{Er}$ ist zytologisch gut darstellbar und im Moment noch sensibel gegen Antimykotika (Clotrimazol, Nystatin, Miconazol). Ziel einer Therapie ist die Wiederherstellung physiologischer Zustände. In der Regel werden dazu Kombinationspräparate verwendet, die ein Glukokortikosteroid enthalten. Sie verbessern die Symptome Erythem, Cerumen, Pruritus und Schwellung besser, als Präparate, die lediglich antimikrobiell wirksam sind. Das Wachstum von opportunistisch pathogenen Keimen muss unterbunden werden. Es ist darauf zu achten, dass der Gehörgang ein gewisses minimales Lumen aufweist und die Medikamente einen direkten Kontakt mit der Haut erhalten. Es sind nur wenige kombinierte Otologika für den Hund verfügbar, die einen oder mehrere pflanzliche Wirkstoffe enthalten. Der Beitrag des Phytotherapeutikums ist jeweils unklar. Immerhin gibt es Hinweise, dass ätherische Öle einen synergistischen Einfluss auf die Wirksamkeit von Clotrimazol haben und dass sie keimhemmend sein können.

\section{Teil 2}

In der kommenden Ausgabe beschäftigen sich die Beiträge u.a. mit Tinnitus und Hyperakusis, dem laryngopharyngealen Reflux und ätherischen Ölen bei Erkältungskrankheiten.

\section{Dank}

Wir danken den Referierenden, dass sie ihre Vortragskurzfassungen für diesen Bericht zur Verfügung gestellt haben.

Eine Dokumentation zur Tagung ist im Internet zu finden: https: / /www.smgp.c h/smgp/archiv/jahrestagungenf/jahresta gung2019.html

Jean-Michel Jeannin und Prof. Dr. sc. nat. Beat Meier, SMGP. Beat.Meier@zhaw.ch 